\section{Dithiocarbamates: a new class of carbonic anhydrase inhibitors. Crystallographic and kinetic investigations $\dagger$}

\author{
Fabrizio Carta, ${ }^{a}$ Mayank Aggarwal, ${ }^{b}$ Alfonso Maresca, ${ }^{a}$ Andrea Scozzafava, ${ }^{a}$ \\ Robert McKenna ${ }^{* b}$ and Claudiu T. Supuran*a
}

Received 14th October 2011, Accepted 12th December 2011

DOI: $10.1039 / \mathrm{c} 2 \mathrm{cc} 16395 \mathrm{k}$

The zinc enzyme carbonic anhydrase (CA, EC 4.2.1.1) is inhibited by several classes of zinc-binders (sulfonamides, sulfamates, and sulfamides) as well as by compounds which do not interact with the metal ion (phenols, polyamines and coumarins). Here we report a new class of potent $\mathrm{CA}$ inhibitors which bind the zinc ion: the dithiocarbamates (DTCs). They coordinate to the zinc ion from the enzyme active site in monodentate manner and establish many favorable interactions with amino acid residues nearby. Several low nanomolar CA I, II and IX inhibitors were detected.

Carbonic anhydrases (CAs, EC 4.2.1.1) are widespread metalloenzymes found in higher vertebrates including humans. 16 isozymes have been characterised to date, many of which are involved in critical physiological processes. ${ }^{1,2}$ They catalyse the following reaction: $\mathrm{CO}_{2}+\mathrm{H}_{2} \mathrm{O}=\mathrm{H}^{+}+\mathrm{HCO}_{3}{ }^{-}$. In humans, CAs are present in a large variety of tissues (gastrointestinal and reproductive tract, nervous system, kidneys, lungs, skin and eyes). ${ }^{1-4}$ The different isozymes are localized in various parts of the cell with CA I and CA II, important isozymes in normal cells, being localised in the cytosol. ${ }^{1,2}$ Many of the CA isozymes are important therapeutic targets with the potential to be inhibited to treat a range of disorders. ${ }^{1,2}$ The classical CA inhibitors (CAIs) are the primary sulfonamides, $\mathrm{RSO}_{2} \mathrm{NH}_{2}$ (and their isosteres, the sulfamates/sulfamides) which are in clinical use for more than 50 years as diuretics and systemically acting antiglaucoma drugs. ${ }^{1,2}$ Apart from their use as diuretics and antiglaucoma agents, recently it has emerged that they have potential as anticonvulsant, antiobesity, anticancer, antipain and antiinfective drugs. ${ }^{1,2}$ Critical barriers to the design of CA inhibitors (CAIs) as therapeutic agents are related to the high number of isoforms present in mammals (i.e., $16 \mathrm{CAs}$, of which 13 have catalytic activity), their diffuse localization in many tissues/organs, and the lack of isozyme selectivity of many of the presently available inhibitors of the sulfonamide/sulfamate type. ${ }^{3-7}$

${ }^{a}$ Università degli Studi di Firenze, Laboratorio di Chimica Bioinorganica, Rm. 188, Via della Lastruccia 3, I-50019 Sesto Fiorentino (Firenze), Italy. E-mail: claudiu.supuran@unifi.it

${ }^{b}$ Department of Biochemistry and Molecular Biology, College of Medicine, University of Florida, Box 100245, Gainesville, Florida 32610, USA. E-mail: rmckenna@ufl.edu; Fax: +1 352 392-3422; Tel: $+1352392-5696$

$\dagger$ Electronic supplementary information (ESI) available. See DOI: $10.1039 / \mathrm{c} 2 \mathrm{cc} 16395 \mathrm{k}$
However, crucial advances have been ultimately made in this field, with several novel chemotypes reported to behave as potent CAIs, ${ }^{3,4,7-10}$ such as the coumarins and thiocoumarins, ${ }^{3,4}$ the polyamines, ${ }^{6}$ etc.

There are two main CA inhibition mechanisms: sulfonamides and their bioisosteres coordinate directly to the metal ion from the enzyme active site whereas phenols, polyamines and coumarins/ thiocoumarins do not directly interact with the zinc ion. ${ }^{1-4}$

All the zinc binders known so far (organic compounds) coordinate monodentately to the zinc ion through a nitrogen atom from the zinc-binding group $(\mathrm{ZBG}),{ }^{7-10}$ whereas few bidentate inhibitors have been discovered, such as a thiomalthol anlogue (HOPTO) ${ }^{11}$ and $N$-hydroxyurea ${ }^{12}$ which were shown to coordinate bidentately to the $\mathrm{Zn}$ (II) ion from the enzyme active site. Dithiocarbamates (DTCs) are well known metal complexing agents and they also possess interesting biomedical and agricultural applications. ${ }^{13,14}$ However few studies investigated their interactions with metalloenzymes. ${ }^{15}$ One such work has investigated the inhibition of $N, N$-diethyl-DTC with bovine CA. ${ }^{16}$ By using Co(II)-substituted CA, Morpurgo et al. ${ }^{16}$ showed that the inhibitor does not extrude the metal ion from the enzyme active site and that it binds to it in a trigonal-bipyramidal geometry of the Co(II) ion. No other DTCs were subsequently investigated for their interaction with $\mathrm{CAs}$ till our group reported that trithiocarbonate, which contains a new $\mathrm{ZBG},\left(\mathrm{CS}_{2}{ }^{-}\right)$as well as diethyl-DTC, inhibit several CA isoforms in the low micromolar or submicromolar range. ${ }^{17}$ Here we extend those findings, showing that a wide range of DTCs incorporating various aliphatic and/or aromatic moieties at the nitrogen atom, act as low nanomolar and even subnanomolar CAIs. We elucidate the mechanism of action of this new class of potent CAIs, by reporting the X-ray crystal structures for the adducts of two DTCs in complex with the human (h) isoform CA II. The investigated DTCs 1-9 were prepared as described in the ESI $\dagger$, by reacting amines with $\mathrm{CS}_{2}$ in the presence of sodium hydroxide.

Three CA isoforms were included in this study (Table 1): the cytosolic CA I and II and the transmembrane, tumor-associated CA IX. CA I and II are the most abundant CAs in the body being also drug targets. ${ }^{1,2}$ CA IX is an anticancer drug target. ${ }^{4,18,19}$

Data of Table 1 show the following interesting findings. Against the slow cytosolic isoform hCA I the activity range of DTCs 1-9 was between $0.97 \mathrm{nM}-1.84 \mu \mathrm{M}$. The simple 
Table 1 CA inhibition data with DTCs 1-9 (R1R2NCS $\left.{ }_{2}^{-} \mathrm{Na}^{+}\right)$and acetazolamide $\mathbf{1 0}$ (as standard inhibitor) against the cytosolic isoforms hCA I and II and transmembrane, tumor-associated one hCA IX

\begin{tabular}{llll}
\hline DTC, $\left(\mathrm{R}^{1}, \mathrm{R}^{2}\right.$ groups $)$ & $\begin{array}{l}K_{\mathrm{I}}(\mathrm{hCA} \mathrm{I}) / \\
\mathrm{nM}\end{array}$ & $\begin{array}{l}K_{\mathrm{I}}(\mathrm{hCA} \text { II }) / \\
\mathrm{nM}\end{array}$ & $\begin{array}{l}K_{\mathrm{I}}(\mathrm{hCA} \text { IX) } \\
\mathrm{nM}\end{array}$ \\
\hline $\mathbf{1}(\mathrm{Me}, \mathrm{Me})$ & 699 & 6910 & 714 \\
$\mathbf{2}(\mathrm{Et}, \mathrm{Et})$ & 790 & 3100 & 1413 \\
$\mathbf{3}(\mathrm{nPr}, \mathrm{nPr})$ & 1838 & 55.5 & 53.8 \\
$\mathbf{4}(\mathrm{nBu}, \mathrm{nBu})$ & 43.1 & 50.9 & 50.3 \\
$\mathbf{5}(\mathrm{iBu}, \mathrm{iBu})$ & 0.97 & 0.95 & 4.5 \\
$\mathbf{6}(\mathrm{Et}, \mathrm{nBu})$ & 157 & 27.8 & 25.9 \\
$\mathbf{7}(\mathrm{Me}, \mathrm{Ph})$ & 39.6 & 21.5 & 28.2 \\
$\left.\mathbf{8}(\mathrm{Me}, \mathrm{PhCH})_{2}\right)$ & 69.9 & 25.4 & 53.0 \\
$\mathbf{9}\left(\mathrm{NC}(\mathrm{Ph}) \mathrm{C}_{2}\left(\mathrm{CH}_{2} \mathrm{CH}_{2}\right)_{2}\right.$ & 48.4 & 40.8 & 757 \\
$\mathbf{1 0}($ acetazolamide $)$ & 250 & 12 & 25
\end{tabular}

Errors were in the range of $5-10 \%$ of the reported data (from 3 different assays, data not shown) by a stopped-flow assay. ${ }^{20}$

dimethyl-DTC was a moderate hCA I inhibitor $\left(K_{\mathrm{I}}\right.$ of $\left.0.7 \mu \mathrm{M}\right)$ and this trend was observed also for its simple congeners incorporating two Et or two $n$-Pr moieties (2 and 3) which showed inhibition constants in the range of $0.79-1.84 \mu \mathrm{M}$. A further elongation of the aliphatic chain led to very strong CAIs, with the $n$-Bu derivative $\mathbf{4}$ being a nanomolar and the iso-butyl isomer $\mathbf{5}$ a subnanomolar hCA I inhibitor (Table 1). The remaining derivatives $\mathbf{6}-\mathbf{9}$, possessing various $\mathrm{R}$ moieties substituting the nitrogen atom showed effective hCA I inhibitory activity, with $K_{\mathrm{I}} \mathrm{s}$ in the range of $39.6-157 \mathrm{nM}$, all these compounds being better CAIs compared to the clinically used sulfonamide acetazolamide 10 (5-acetamido-1,3,4-thiadiazole-2sulfonamide). ${ }^{1}$

For the rapid isoform hCA II a different inhibition pattern has been observed. Thus, the simplest derivatives $\mathbf{1}$ and $\mathbf{2}$ behaved as ineffective CAIs, with $K_{\mathrm{I}}$ in the range of 3.1-6.9 $\mu \mathrm{M}$, whereas all the remaining DTCs investigated here showed highly effective such properties, with $K_{\mathrm{I}} \mathrm{s}$ in the range of $0.95-55.5 \mathrm{nM}$. The most effective inhibitor was again the di-isobutyl-DTC 5 .

The tumor-associated extracellular isoform hCA IX was also inhibited by all these compounds, with $K_{\mathrm{I}}$ in the range of 4.5-1413 nM (Table 1). The simple aliphatic derivatives $\mathbf{1}$ and $\mathbf{2}$, as well as the piperidine derivative $\mathbf{9}$ were ineffective CAIs ( $K_{\mathrm{I}} \mathrm{s}$ of $714-1413 \mathrm{nM}$ ) whereas the remaining compounds showed a highly enhanced efficacy $\left(K_{\mathrm{I}} \mathrm{S}\right.$ in the range of $\left.4.5-53.8 \mathrm{nM}\right)$, being more effective compared to the sulfonamide $\mathbf{1 0}$ as inhibitors of hCA IX.

These data prove that small structural changes in the molecule of this new class of CAIs lead to dramatic changes of the enzyme inhibitory activity, which is an interesting finding when a new class of inhibitors is reported. In order to rationalize these findings at the molecular level, we resolved the X-ray crystal structure for the adducts of two of these DTCs bound to hCA II. ${ }^{21}$

The X-ray crystal structures of CA II complexed with $\mathbf{8}$ and 9 have been determined (Fig. 1) to $1.5 \AA$ resolution, using a protocol as previously described by us (refer to supplementary text and Table S1 (ESI $\dagger$ ) for experimental details). ${ }^{9}$ The two compounds were well ordered and refined with occupancies of 0.85 and 1.00 and B-factors that were comparable to the solvent within the active site. The hydrophobic non-planar compounds are buried deep into the active site, displacing the
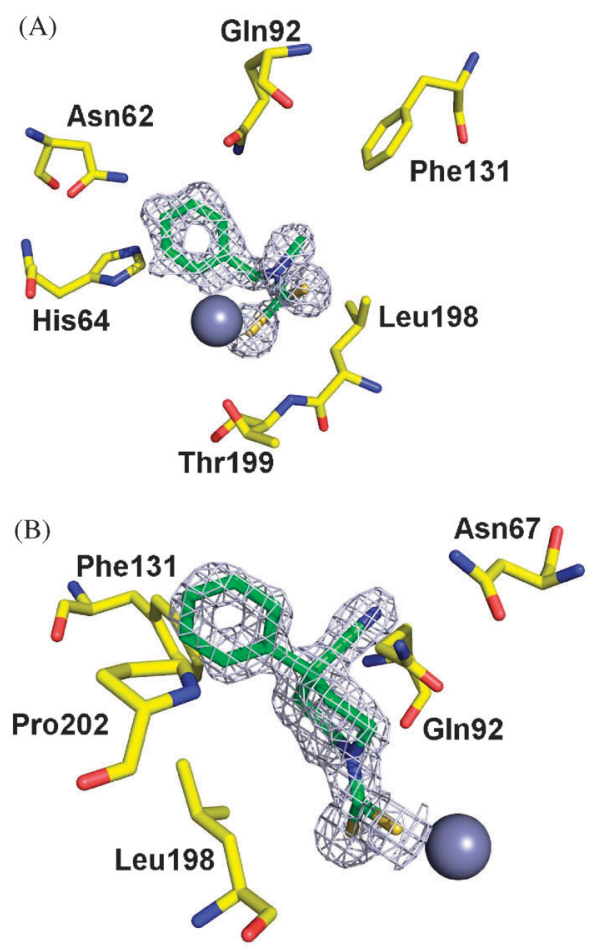

Fig. 1 Electron densities for X-ray crystal structures of adducts of DTCs 8 (A) and 9 (B) with hCA II.

catalytic zinc-bound solvent, such that one of the sulfurs binds directly to the zinc atom of hCA II (distance $\sim 2.3 \AA$ ). Hence the overall $\mathrm{Zn}$ ( $3 \mathrm{~N}$ from coordinating histidines, and $1 \mathrm{~S}$ inhibitor ligand) coordination can be described as a distorted tetrahedron. Both compounds protrude out of the active site with their tail groups stabilized predominantly by hydrophobic residues. Compound $\mathbf{8}$ exhibits van der Waals interactions with the side chains of Asn62, His64, Glu92, His94, Val121, Phe131, Leu198, Thr200 and Pro202 (Fig. 1A and 2 (green)), with an average B-factor of $20.0 \AA^{2}$ and occupancy of 0.85 (Table S1, ESI $\dagger$ ). Approximately $330 \AA^{2}(92 \%)$ of the surface area of the compound is buried. Compound $\mathbf{9}$, the largest in size among the three compounds, possesses a nitrogen atom which hydrogen bonds with a surrounding water molecule and to the side chains of Asn67 and Gln92, and as a consequence buries $\sim 360 \AA^{2}$ $(82.3 \%)$ of its surface area. The hydrophobic cyclohexane ring at the distal end of the compound protrudes outwards from the active site and is additionally stabilized by the side chains of Phe131, Leu 198 and Pro202 (Fig. 1B and 2 (pink)).

In conclusion we report here that the $N, N$-disubstituted dithiocarbamates inhibit various CA isoforms (established drug targets for a variety of disorders) with efficiencies between the subnanomolar to the micromolar, depending on the nature of the aliphatic/aromatic groups present in their molecules. By means of high resolution X-ray crystallography we also explain the good efficacy of this new class of CAIs to inhibit various isoforms. Due to their simple chemical nature, ease of preparation and good water solubility, DTCs may show various applications in fields in which CA inhibition is necessary.

This research was financed by an FP7 EU project (Metoxia) and a grant from the NIH GM 25154. 


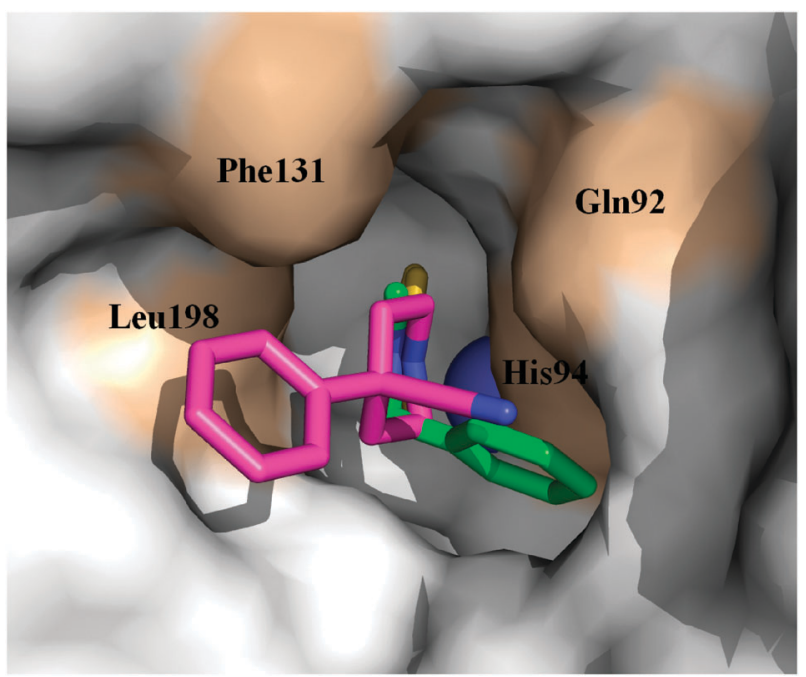

Fig. 2 View of compounds 8 (green), 9 (magenta), superposed in the active site of hCA II. hCA II is depicted as a grey surface representation. Amino acids labeled surfaces are colored salmon. The active-site zinc is depicted as a blue sphere. This figure has been illustrated using PyMOL (DeLano Scientific).

\section{Notes and references}

1 (a) C. T. Supuran, Nat. Rev. Drug Discovery, 2008, 7, 168; (b) C. T. Supuran, Bioorg. Med. Chem. Lett., 2010, 20, 346.

2 (a) C. T. Supuran, A. Scozzafava and A. Casini, Med. Res. Rev., 2003, 23, 146; (b) C. T. Supuran, Curr. Pharm. Des., 2010, 16, 3233.

3 (a) A. Maresca, C. Temperini, H. Vu, N. B. Pham, S. A. Poulsen, A. Scozzafava, R. J. Quinn and C. T. Supuran, J. Am. Chem. Soc., 2009, 131, 3057; (b) A. Maresca, C. Temperini, L. Pochet, B. Masereel, A. Scozzafava and C. T. Supuran, J. Med. Chem., 2010, 53, 335.

4 D. Neri and C. T. Supuran, Nat. Rev. Drug Discovery, 2011, 10, 767.

5 P. Ebbesen, E. O. Pettersen, T. A. Gorr, G. Jobst, K. Williams, J. Kienninger, R. H. Wenger, S. Pastorekova, L. Dubois, P. Lambin, B. G. Wouters, C. T. Supuran, L. Poellinger, P. Ratcliffe, A. Kanopka, A. Görlach, M. Gasmann, A. L. Harris, P. Maxwell and A. Scozzafava, J. Enzyme Inhib. Med. Chem., 2009, 24(S1), 1.

6 (a) F. Carta, C. Temperini, A. Innocenti, A. Scozzafava, K. Kaila and C. T. Supuran, J. Med. Chem., 2010, 53, 5511; (b) A. Maresca, A. Scozzafava and C. T. Supuran, Bioorg. Med. Chem. Lett., 2010, 20, 7255; (c) A. Maresca and C. T. Supuran, Bioorg. Med. Chem. Lett., 2010, 20, 4511.

7 V. Alterio, A. Di Fiore, K. D'Ambrosio, C. T. Supuran and G. De Simone, X-Ray crystallography of CA inhibitors and its importance in drug design, in Drug Design of Zinc-Enzyme Inhibitors: Functional, Structural, and Disease Applications, ed. C. T. Supuran and J. Y. Winum, Wiley, Hoboken, 2009, pp. 73-138.

8 (a) F. Pacchiano, M. Aggarwal, B. S. Avvaru, A. H. Robbins, A. Scozzafava, R. McKenna and C. T. Supuran, Chem. Commun., 2010, 46, 8371; (b) A. Di Fiore, S. M. Monti, M. Hilvo, S. Parkkila, V. Romano, A. Scaloni, C. Pedone, A. Scozzafava, C. T. Supuran and G. De Simone, Proteins, 2009, 74, 164.

9 (a) B. S. Avvaru, J. M. Wagner, A. Maresca, A. Scozzafava, A. H. Robbins, C. T. Supuran and R. McKenna, Bioorg. Med.
Chem. Lett., 2010, 20, 4376; (b) J. M. Wagner, B. S. Avvaru, A. H. Robbins, A. Scozzafava, C. T. Supuran and R. McKenna, Bioorg. Med. Chem., 2010, 18, 4873.

10 (a) J. F. Domsic, B. S. Avvaru, C. U. Kim, S. M. Gruner, M. Agbandje-McKenna, D. N. Silverman and R. McKenna, J. Biol. Chem., 2008, 283, 30766; (b) D. W. Christianson and C. A. Fierke, Acc. Chem. Res., 1996, 29, 331; (c) K. Köhler, A. Hillebrecht, J. Schulze Wischeler, A. Innocenti, A. Heine, C. T. Supuran and G. Klebe, Angew. Chem., Int. Ed., 2007, 46, 7697; (d) A. E. Eriksson, T. A. Jones and A. Liljas, Proteins, 1988, 4, 274.

11 J. Schulze Wischeler, A. Innocenti, D. Vullo, A. Agrawal, S. M. Cohen, A. Heine, C. T. Supuran and G. Klebe, ChemMedChem, 2010, 5, 1609.

12 C. Temperini, A. Innocenti, A. Scozzafava and C. T. Supuran, Bioorg. Med. Chem. Lett., 2006, 16, 4316.

13 A. Bencini, P. Failli, B. Valtancoli and D. Bani, Cardiovasc. Hematol. Agents Med. Chem., 2010, 8, 128.

14 O. H. Szolar, Anal. Chim. Acta, 2007, 582, 191.

15 E. Amin, A. A. Saboury, H. Mansouri-Torshizi and A. A. MoosaviMovahedi, J. Enzyme Inhib. Med. Chem., 2010, 25, 272.

16 L. Morpurgo, A. Desideri, A. Rigo, P. Viglino and G. Rotilio, Biochim. Biophys. Acta, 1983, 746, 168.

17 (a) A. Innocenti, A. Scozzafava and C. T. Supuran, Bioorg. Med. Chem. Lett., 2009, 19, 1855; (b) A. Innocenti, A. Scozzafava and C. T. Supuran, Bioorg. Med. Chem. Lett., 2010, 20, 1548; (c) C. Temperini, A. Scozzafava and C. T. Supuran, Bioorg. Med. Chem. Lett., 2010, 20, 474.

18 Y. Lou, P. C. McDonald, A. Oloumi, S. Chia, C. Oslund, A. Ahmadi, A. Kyle, U. auf dem Keller, S. Leung, D. Huntsman, B. Clarke, B. W. Sutherland, A. D. Waterhouse, M. Bally, C. Roskelley, C. M. Overall, A. Minchinton, F. Pacchiano, F. Carta, A. Scozzafava, N. Touisni, J. Y. Winum, C. T. Supuran and S. Dedhar, Cancer Res., 2011, 71, 3364.

19 V. Alterio, M. Hilvo, A. Di Fiore, C. T. Supuran, P. Pan, S. Parkkila, A. Scaloni, J. Pastorek, S. Pastorekova, C. Pedone, A. Scozzafava, S. M. Monti and G. De Simone, Proc. Natl. Acad. Sci. U. S. A., 2009, 106, 16233.

20 R. G. Khalifah, J. Biol. Chem., 1971, 246, 2561. An applied photophysics stopped-flow instrument has been used for assaying the CA catalysed $\mathrm{CO}_{2}$ hydration activity. Phenol red (at a concentration of $0.2 \mathrm{mM}$ ) has been used as indicator, working at the absorbance maximum of $557 \mathrm{~nm}$, with $20 \mathrm{mM}$ HEPES (pH 7.5) as buffer, and $20 \mathrm{mM} \mathrm{Na}_{2} \mathrm{SO}_{4}$ (for maintaining constant the ionic strength), following the initial rates of the CA-catalyzed $\mathrm{CO}_{2}$ hydration reaction for a period of $10-100 \mathrm{~s}$. Stock solutions of an inhibitor $(0.1 \mathrm{mM})$ were prepared in distilled-deionized water and dilutions up to $0.01 \mathrm{nM}$ were done thereafter with distilled-deionized water. The inhibition constants were obtained by non-linear least-squares methods ${ }^{6}$.

21 Co-crystals of the two CA II complexes were obtained using the hanging drop vapor diffusion method. Drops of $10 \mu \mathrm{L}(0.3 \mathrm{mM}$ hCA II; $0.7 \mathrm{mM}$ drug; $0.1 \%$ dimethyl sulfoxide (DMSO); $0.8 \mathrm{M}$ sodium citrate; $50 \mathrm{mM}$ Tris- $\mathrm{HCl}$; $\mathrm{pH} 8.0$ ) were equilibrated against the precipitant solution (1.6 M sodium citrate; $50 \mathrm{mM}$ Tris- $\mathrm{HCl}$; $\mathrm{pH}$ 8.0) at room temperature. Crystals were observed after 5 days. Crystals were cryoprotected by immersion into $20 \%$ glycerol precipitant solution and flash-cooled at $100 \mathrm{~K}$. The X-ray diffraction data were collected using an R-AXIS $\mathrm{IV}^{++}$image plate system on a Rigaku RU-H3R Cu rotating anode operating at $50 \mathrm{kV}$ and $22 \mathrm{~mA}$, using Osmic Varimax HR optics. The detector-crystal distance was set to $80 \mathrm{~mm}$. The oscillation steps were $1^{\circ}$ with a 5 min exposure per image. Indexing, integration, and scaling were performed using HKL2000. 